\title{
RELAÇÃO ENTRE O TEMPO E O TIPO DE AMAMENTAÇÃO E AS FUNÇÕES DO SISTEMA ESTOMATOGNÁTICO
}

\section{Relationship between time and type of breastfeeding and stomatogna- thic system functions}

\author{
Aline Prade Neu (1), Ana Maria Toniolo da Silva (2), Carolina Lisbôa Mezzomo (3), \\ Angela Ruviaro Busanello-Stella ${ }^{(4)}$, Anaelena Bragança de Moraes ${ }^{(5)}$
}

\section{RESUMO}

Objetivo: investigar a relação do tempo e tipo de aleitamento com as funções de mastigação, deglutição e respiração. Método: a amostra foi constituída por 52 crianças de ambos os sexos, na faixa etária de 5 a 8 anos. Utilizando-se de questionário, foram coletados dados referentes ao tipo e tempo de amamentação e respiração das crianças. As mesmas foram submetidas à avaliação clínica das funções, a qual baseou-se no Protocolo de Avaliação Miofuncional Orofacial com Escores Expandido (AMIOFE- E). Os dados foram analisados de forma descritiva e estatística. Para testar a normalidade da variável quantitativa, utilizou-se o teste Lilliefors. Todas as relações entre variáveis foram realizadas por meio do teste Qui-quadrado, considerando nível de significância de 5\%. Resultados: não houve relação estatisticamente significante entre o tempo e o tipo de aleitamento e as variáveis respiração e deglutição. Encontrou-se significância estatística entre tipo de aleitamento e a mastigação, mas não entre o tempo de aleitamento e esta variável. Além disso, a relação significante não seguiu padrão esperado. Conclusão: foi possível concluir que, de um modo geral, não houve relação significante entre o tempo e o tipo de aleitamento com as funções estudadas, exceto pela significância entre o tipo de aleitamento e a mastigação. Provavelmente a falta de significância deva-se ao fato do desenvolvimento de todo o sistema estomatognático ter origem multifatorial. A significância estatística encontrada para a função de mastigação, visto que foi com padrão inesperado, pode ter ocorrido em virtude do restrito número de sujeitos em cada subgrupo.

DESCRITORES: Sistema Estomatognático; Respiração Bucal; Amamentação; Mastigação; Deglutição

(1) Fonoaudióloga; Mestranda do Programa de Pós-Graduação em Distúrbios da Comunicação Humana da Universidade Federal de Santa Maria, UFSM, Santa Maria, RS, Brasil.

(2) Fonoaudióloga; Professora Adjunta da Universidade Federal de Santa Maria, UFSM, Santa Maria, RS, Brasil; Doutora em Distúrbios da Comunicação Humana pela Universidade Federal de São Paulo.

(3) Fonoaudióloga; Professora Adjunta da Universidade Federal de Santa Maria, UFSM, Santa Maria, RS, Brasil; Doutora em Letras pela Pontifícia Universidade Católica do Rio Grande do Sul.

(4) Fonoaudióloga;Doutoranda do Programa de Pós-Graduação em Distúrbios da Comunicação Humana da Universidade Federal de Santa Maria, UFSM, Santa Maria, RS, Brasil.

(5) Professora Adjunta da Universidade Federal de Santa Maria, UFSM, Santa Maria, RS, Brasil; Doutora em Epidemiologia pela Universidade Federal do Rio Grande do Sul, Porto Alegre, RS, Brasil.

Conflito de interesses: inexistente

\section{INTRODUÇÃO}

O controle motor orofacial do bebê evolui de movimentos instáveis e indiferenciados para um padrão de equilíbrio, onde se observam estabilidade e diferenciação de suas estruturas. Este equilíbrio será influenciado pela maturação do sistema nervoso, do crescimento músculo-esquelético e das experiências sensoriais e motoras vivenciadas pelo recém-nascido'.

Entre estas experiências, destaca-se o aleitamento natural, pois a sucção que o bebê realiza na mama irá possibilitar o adequado desenvolvimento do sistema estomatognático, favorecendo a maturação normal de todo o complexo craniofacial $^{2}$. Quando a amamentação natural mostra-se inviável, vias alternativas de alimentação tornam-se presentes, dentre elas a mamadeira ${ }^{3}$. 
Sabe-se que, o leite materno deve ser exclusivo desde o nascimento até os seis meses de idade, sendo complementado a partir de então até os dois anos ou mais ${ }^{4-7}$. A sua interrupção ou a introdução e manutenção do aleitamento artificial por um longo período, podem gerar alterações estruturais e funcionais no sistema estomatognático ${ }^{8}$. De modo geral, estas alterações serão influenciadas pela tríade; reduzido tempo de aleitamento materno, associado ao período prolongado do uso da mamadeira e a frequência com que esta é utilizada ${ }^{3}$.

Assim como o desmame precoce, a amamentação artificial pode interferir negativamente sobre o desenvolvimento adequado da criança, incluindo as estruturas e funções orofaciais ${ }^{8,9}$. Além disso, o uso da mamadeira pode incentivar o surgimento de outros hábitos orais deletérios, uma vez que, a criança não supre suas necessidades de sucção e acaba adquirindo hábitos de sucção não nutritiva ${ }^{10}$.

Partindo-se dos aspectos vistos acima, o objetivo do presente estudo foi investigar a relação do tempo e tipo de aleitamento com as funções de mastigação, deglutição e respiração.

\section{MÉTODO}

Esta pesquisa foi realizada com crianças de oito escolas públicas e particulares do município de Agudo - Rio Grande do Sul. A amostra foi constituída por participantes, de ambos os sexos, na faixa etária entre cinco anos e 8 anos e 9 meses. Os mesmos assentiram sua participação na pesquisa e seus responsáveis assinaram o Termo de Consentimento Livre e Esclarecido (TCLE). Após a adesão ao termo, os responsáveis de 141 crianças preencheram um questionário previamente elaborado pelas autoras.

O critério de inclusão deste estudo foi estar na faixa etária entre cinco anos e 8 anos e 9 meses de idade. As crianças que apresentaram sinais evidentes de comprometimento neurológico, cognitivo ou emocional grave e/ou cujos pais responderam o questionário de forma indevida foram excluídas da pesquisa. Assim, 52 crianças contemplaram os critérios do estudo.

No que se refere ao questionário, foram coletados dados de identificação das crianças, referentes ao tipo e tempo de amamentação recebidos e à respiração.

Quanto ao tipo de aleitamento, os indivíduos da amostra foram distribuídos em três categorias: peito exclusivo; mamadeira exclusiva; ou misto, quando os dois foram ofertados. Quanto ao tempo de amamentação, foram consideradas também 3 categorias para aleitamento natural e artificial: nenhum ou pouco, quando a criança foi amamentada até
3 meses de idade; até 2 anos, quando a criança recebeu aleitamento entre 4 meses e 2 anos de idade; e além de 2 anos, quando foi ofertado aleitamento além desta idade.

As questões sobre respiração (4 itens) referiram-se ao ronco e à baba noturnos; à permanência de lábios entreabertos/abertos durante o dia e quanto à presença de garganta seca ao acordar. As crianças foram consideradas respiradoras orais, nesta etapa, se apresentassem pelo menos três itens indicativos de tal patologia.

As mesmas foram submetidas à avaliação clínica, sendo avaliadas individualmente pelo mesmo examinador, utilizando o Protocolo de Avaliação Miofuncional Orofacial com Escores Expandido (AMIOFE- E) ${ }^{11}$, adaptado para esta pesquisa. A avaliação foi realizada por inspeção visual, durante a sessão e complementada pela análise posterior das filmagens realizadas durante a avaliação. Os indivíduos permaneceram sentados em cadeira com encosto e os pés apoiados, sendo adotadas regras de padronização de distância entre estes e a lente $(60 \mathrm{~cm})$ e a altura da mesa (focou-se a altura dos olhos da criança).

$\mathrm{Na}$ avaliação clínica, foram coletados dados referentes às funções de mastigação, deglutição e respiração. Para avaliar a mastigação, solicitou-se que as crianças mastigassem três pedaços de pão francês de modo habitual. Considerou-se padrão mastigatório normal, quando ocorreu mastigação bilateral alternada; e padrão mastigatório alterado quando houve mastigação bilateral simultânea, mastigação unilateral preferencial, mastigação unilateral crônica ou mastigação anterior.

Para a avaliação da deglutição, as crianças foram orientadas a tomar água de forma habitual (vários goles seguidos). Observou-se também a deglutição do pão francês. Desta forma, foram analisados os seguintes itens: vedamento labial sem esforço, interposição lingual, movimento de cabeça ou outras partes do corpo, tensão do músculo mentual, tensão do músculo orbicular da boca, escape de alimento e ruído. A deglutição foi considerada alterada, quando as crianças apresentaram pelo menos três destes componentes alterados.

A respiração foi avaliada clinicamente por meio de observação durante conversa informal com as crianças e do teste da água. Na observação, foi analisado se as mesmas mantinham os lábios entreabertos ou abertos a maior parte do tempo, o que era interpretado como indício de respiração alterada. No teste da água, foi solicitado, que fosse colocado água na boca e permanecessem com os lábios em contato, sem engolir pelo menos por 3 minutos $^{12}$. Aquelas que não conseguissem manter por este período os lábios em contato, tendo que 
engolir a água, foram consideradas como respiradoras orais.

Assim, para a criança ser considerada respiradora oral, deveria apresentar, em pelo menos 2 itens dos que seguem, confirmação de alteração nesta função: questionário, inspeção visual e teste da água.

Este estudo foi aprovado pelo Comitê de Ética em Pesquisa da Universidade Federal de Santa Maria - CEP/UFSM, sob o protocolo de número 0223.0.243.000-10.

As variáveis estudadas foram analisadas de forma descritiva e estatística. O programa Statistica 9.0 foi utilizado para a análise estatística dos dados.

Inicialmente foi calculada a normalidade utilizando-se o Teste Lilliefors para a variável quantitativa "tempo de amamentação". Esta variável não seguiu comportamento normal e, por este motivo, foi realizado teste não paramétrico Qui-Quadrado para relação com as variáveis referentes à deglutição, respiração e mastigação. A relação entre as variáveis "tipo de aleitamento" e as demais (todas qualitativas) também foi realizada pelo Teste Qui-Quadrado. Nas análises estatísticas foi utilizado nível de significância de $5 \%(p<0,05)$.

\section{RESULTADOS}

Observam-se os dados descritivos referentes ao tipo de amamentação, tempo de aleitamento materno, tempo de aleitamento artificial, respiração, deglutição e mastigação.

O tipo de aleitamento foi relacionado com as variáveis respiração, deglutição e mastigação.

O tempo de aleitamento natural e artificial e as funções de respiração, deglutição e mastigação também foram relacionados neste estudo.

Tabela 1 - Distribuição dos valores absolutos (n) e relativos (\%) das variáveis, tipo de amamentação, tempo de aleitamento natural, tempo de aleitamento artificial, respiração, deglutição e mastigação

\begin{tabular}{cccc}
\hline & Variáveis & $\mathbf{n}$ & $\%$ \\
\hline \multirow{2}{*}{ Tipo amamentação } & Peito & 10 & 19,23 \\
& Mamadeira & 10 & 19,23 \\
& Misto & 32 & 61,54 \\
\hline \multirow{3}{*}{ Tempo Aleitamento Natural } & Nenhum ou pouco & 21 & 40,38 \\
& Até 2 anos & 20 & 38,47 \\
& Além 2 anos & 11 & 21,15 \\
\hline \multirow{3}{*}{ Tempo Aleitamento Artificial } & Nenhum ou pouco & 10 & 19,23 \\
& Até 2 anos & 5 & 9,62 \\
& Além 2 anos & 37 & 71,15 \\
\hline \multirow{2}{*}{ Respiração } & Modo Nasal & 39 & 75,00 \\
& Modo Oral & 13 & 25,00 \\
\hline \multirow{2}{*}{ Deglutição } & Normal & 35 & 67,30 \\
& Alterada & 17 & 32,70 \\
\hline \multirow{2}{*}{ Mastigação } & Normal & 24 & 46,15 \\
& Alterada & 28 & 53,85 \\
\hline
\end{tabular}

Tabela 2 - Distribuição da relação entre tipo de aleitamento e as variáveis respiração, deglutição e mastigação (valores absolutos e percentuais)

\begin{tabular}{lcccccc}
\hline \multirow{2}{*}{$\begin{array}{l}\text { Tipo } \\
\text { Aleitamento }\end{array}$} & \multicolumn{2}{c}{ Modo Respiratório } & \multicolumn{2}{c}{ Deglutição } & \multicolumn{2}{c}{ Mastigação } \\
\cline { 2 - 7 } & Nasal (\%) & Oral (\%) & Normal (\%) & Alterada (\%) & Normal (\%) & Alterada (\%) \\
\hline Peito & $8(80,0)$ & $2(20,0)$ & $7(70,0)$ & $3(30,0)$ & $2(20,0)$ & $8(80,0)$ \\
Mamadeira & $7(70,0)$ & $3(30,0)$ & $9(90,0)$ & $1(10,0)$ & $3(30,0)$ & $7(70,0)$ \\
Misto & $24(75,0)$ & $8(25,0)$ & $19(59,4)$ & $13(40,6)$ & $19(59,4)$ & $13(40,6)$ \\
\hline p-valor & \multicolumn{2}{c}{0,875} & \multicolumn{2}{c}{0,193} & \multicolumn{2}{c}{$0,048^{*}$} \\
\hline
\end{tabular}

* Significância Estatística pelo Teste Qui-Quadrado 
Tabela 3 - Distribuição da relação entre tempo de aleitamento natural e artificial e as variáveis respiração, deglutição e mastigação (valores absolutos e percentuais)

\begin{tabular}{|c|c|c|c|c|c|c|c|}
\hline \multirow{2}{*}{\multicolumn{2}{|c|}{ Tempo Aleitamento }} & \multicolumn{2}{|c|}{ Respiração } & \multicolumn{2}{|c|}{ Deglutição } & \multicolumn{2}{|c|}{ Mastigação } \\
\hline & & Nasal & Oral & Normal & Alterado & Normal & Alterado \\
\hline \multirow{4}{*}{$\begin{array}{c}\text { Tempo } \\
\text { Aleitamento } \\
\text { PT }\end{array}$} & $\begin{array}{l}\text { Nenhum } \\
\text { ou pouco }\end{array}$ & $15(71,4)$ & $6(28,6)$ & $15(71,4)$ & $6(28,6)$ & $8(38,1)$ & $13(61,9)$ \\
\hline & Até 2 anos & $16(80,0)$ & $4(20,0)$ & $14(70,0)$ & $6(30,0)$ & $12(60,0)$ & $8(40,0)$ \\
\hline & $\begin{array}{c}\text { Além } 2 \\
\text { anos }\end{array}$ & $8(72,7)$ & $3(27,3)$ & $6(54,6)$ & $5(45,4)$ & $4(36,4)$ & $7(63,6)$ \\
\hline & p-valor & \multicolumn{2}{|c|}{0,802} & \multicolumn{2}{|c|}{0,593} & \multicolumn{2}{|c|}{0,284} \\
\hline \multirow{4}{*}{$\begin{array}{c}\text { Tempo } \\
\text { Aleitamento } \\
\text { MM }\end{array}$} & $\begin{array}{l}\text { Nenhum } \\
\text { ou pouco }\end{array}$ & $8(80,0)$ & $2(20,0)$ & $7(70,0)$ & $3(30,0)$ & $2(20,0)$ & $8(80,0)$ \\
\hline & Até 2 anos & $4(80,0$ & $1(20,0)$ & $3(60,0)$ & $2(40,0)$ & $2(40,0)$ & $3(60,0)$ \\
\hline & $\begin{array}{l}\text { Além } 2 \\
\text { anos }\end{array}$ & $27(72,9)$ & $10(27,1)$ & $25(67,6)$ & $12(32,4)$ & $20(54,1)$ & $17(45,9)$ \\
\hline & p-valor & \multicolumn{2}{|c|}{0,868} & \multicolumn{2}{|c|}{0,925} & \multicolumn{2}{|c|}{0,152} \\
\hline
\end{tabular}

\section{DISCUSSÃO}

Verificou-se neste estudo que o tipo de aleitamento prevalente foi o misto, pois $61,54 \%$ das crianças foram amamentadas no peito e na mamadeira. Estes dados concordam com outro estudo realizado com 176 crianças de 6 a 12 anos, onde foi verificada alta incidência do aleitamento do tipo misto $(81,82 \%)^{8}$. A interrupção do aleitamento materno e a oferta de outros tipos de leite, com introdução da mamadeira, podem ocorrer devido a uma série de aspectos culturais que estão relacionados à amamentação. Opiniões de que o leite é fraco e insuficiente são algumas das razões para o desmame ${ }^{13}$.

Quanto ao tempo de aleitamento natural, observou-se que a maioria $(40,38 \%$ das crianças) não recebeu aleitamento, ou o recebeu por pouco tempo. Em um estudo semelhante, onde foram investigadas 200 crianças, por meio da aplicação de questionário às mães, verificou-se que $60,05 \%$ das crianças também não receberam amamentação natural, ou receberam por pouco tempo ${ }^{14}$.

Sabe-se que 0 aleitamento materno nos primeiros seis meses de vida é de suma importância para o bebê, uma vez que, considera-se o leite da mãe o melhor alimento para o recém-nascido ${ }^{6}$. Além dos valores nutricionais indiscutíveis que este leite possui, este tipo de amamentação fortalece o vínculo mãe e filho, possibilita o aumento de anticorpos, o ganho de peso para o bebê e proporciona um bom desenvolvimento e fortalecimento das estruturas orais envolvidas no ato de sugar ${ }^{3}$. $O$ desmame precoce pode romper com este desenvolvimento adequado, provocando alterações nas estruturas e funções do sistema estomatognático ${ }^{10}$.

No que se refere ao tempo de aleitamento artificial, constatou-se que $71,15 \%$ das crianças estudadas receberam este tipo de aleitamento por mais de dois anos. Dados semelhantes foram encontrados em outro estudo realizado com 44 crianças de 3 a 5 anos de idade. Os autores observaram que $52,8 \%$ dos sujeitos da pesquisa utilizaram a mamadeira por um período superior a três anos ${ }^{15}$.

Ao extrair o leite da mama, o bebê realiza o movimento de ordenha, o qual exige um grande esforço muscular ${ }^{3}$. Já, quando se usa a mamadeira esta, propicia o trabalho apenas dos músculos bucinadores e do orbicular da boca, deixando de estimular outros músculos importantes, como pterigóideos, masseter, temporal, digástrico, genio-hióideo e milo-hióideo ${ }^{16}$. Deste modo, esta opção de amamentação irá favorecer o surgimento de atipias em todo o sistema estomatognático. Além disso, a amamentação artificial prolongada pode ocasionar o aparecimento de hábitos orais deletérios não-nutritivos que maximizam estas alterações ${ }^{10,8}$.

Tanto a função de respiração, quanto de deglutição, apresentaram-se normais neste estudo. Observou-se que $75,00 \%$ das crianças eram respiradoras nasais e 67,30\% apresentaram deglutição normal. Assim, verificou-se que, nesta pesquisa, os fatores tempo e tipo de amamentação não 
influenciaram no desenvolvimento de alterações, na maioria da amostra.

Entretanto, estes achados divergem dos encontrados na literatura, uma vez que, geralmente, crianças que fazem uso de mamadeira e/ou sofrem desmame precoce, sendo pouco estimuladas quanto às estruturas e funções orais, apresentam alterações como respiração oral ${ }^{17}$ e deglutição alterada $^{18}$.

A respiração, função vital e inata do ser humano, pode sofrer influências negativas do desmame precoce, uma vez que, a criança mantém a posição adequada de repouso de lábios e língua durante o aleitamento no peito, favorecendo a permanência deste padrão correto após o término da amamentação $0^{10,16,19}$. Da mesma forma que a respiração, a deglutição também pode ser influenciada pela interrupção do aleitamento natural, este fato, associado ao uso da mamadeira por período prolongado, pode gerar atipia nesta função, pois estes provocam alterações na musculatura orofacial, na postura das estruturas, na formação da arcada dentária e no palato ${ }^{10}$.

Quanto à mastigação, 53,85\% das crianças deste estudo, apresentaram esta função alterada. Este resultado pode ter relação com o período de dentição no qual a amostra se encontrava, ou seja, período misto de dentição. Segundo a literatura, alterações na função de mastigação podem estar relacionadas a condições morfofuncionais inadequadas, dentre elas o tipo de dentição, tensão e mobilidade de estruturas como lábios, língua e bochechas, entre outros ${ }^{20}$.

De um modo geral não houve associação estatisticamente significante entre as variáveis tempo e tipo de aleitamento com as variáveis respiração, deglutição e mastigação. Apenas a relação tipo de aleitamento com a variável mastigação mostrou-se estatisticamente significante.

Diferentemente do observado nesta pesquisa, os autores de outro estudo com 62 crianças verificaram que as consideradas respiradoras nasais foram amamentadas preferencialmente pelo seio materno nos seis primeiros meses de vida. Em contrapartida, as crianças que não foram amamentadas ou foram, por um período restrito de até três meses de idade, desenvolveram problemas respiratórios, tornando-se respiradoras orais ${ }^{21}$.

Concordando com os resultados encontrados neste estudo, quanto à associação entre aleitamento e deglutição, outros autores em pesquisa realizada com 176 crianças de 6 a 12 anos, não observaram significância estatística entre a relação tempo de aleitamento natural e artificial com a função de deglutição ${ }^{8}$.
Provavelmente a falta de relação direta entre tipo/tempo de aleitamento e as funções de deglutição, respiração e mastigação, observada nesta pesquisa deva-se ao fato de que o desenvolvimento destas funções possua influência de diversos componentes, caracterizando-se como multifatorial, não sendo condicionadas apenas pelo tempo e tipo de aleitamento.

A literatura confirma o fato da respiração oral e das alterações de deglutição estarem associadas a diversas etiologias. O desmame precoce é uma delas, pois este propicia o surgimento de uma posição mais baixa de língua, levando a boca a permanecer entreaberta, favorecendo a respiração oral. Sabe-se também que durante a amamentação no peito, ocorre a participação ativa de diversos músculos, os quais, posteriormente serão fundamentais para a deglutição ${ }^{8,19}$. Além da interrupção precoce da amamentação natural, fatores genéticos como desenvolvimento maxilar, mandibular, de palato e arcada dentária também podem estar associados às alterações das funções do sistema estomatognático ${ }^{22,23}$, assim como os estímulos orais inadequados, dentre eles os hábitos orais deletérios $^{24}$. A respiração oral ainda pode ter etiologias obstrutivas, quando existe algum impedimento mecânico à passagem do fluxo de ar por via nasal ${ }^{25}$, ou viciosas quando decorre de hábitos orais prolongados, alterações musculares, edema transitório da mucosa nasal, entre outros fatores ${ }^{26}$.

Ao analisar a relação entre a variável tipo de aleitamento com a variável mastigação, constatou-se significância estatística. Entretanto, conforme observado na Tabela 2, a relação entre as mesmas não mostrou uma padrão de evolução, seja ele positivo (com a amamentação natural menos as alterações de mastigação) ou negativo (com a amamentação artificial maiores alterações desta função). Este comportamento não previsto pode estar relacionado ao reduzido número de sujeitos em cada subgrupo e, logo, ter influenciado a significância estatística.

Em estudo semelhante observou-se correlações negativas entre a duração do aleitamento artificial e o desempenho mastigatório ${ }^{8}$. Este dado confirma a hipótese de que estímulos inadequados e por longos períodos podem acarretar prejuízos às funções estomatognáticas ${ }^{10}$.

\section{CONCLUSÃO}

Com base no presente estudo, foi possível concluir que, na amostra, de um modo geral, não houve relação significante entre o tempo e o tipo de aleitamento com as funções estudadas, exceto 
pela significância entre o tipo de aleitamento e a mastigação.

Provavelmente a falta de significância deva-se ao fato do desenvolvimento de todo o sistema estomatognático ter origem multifatorial. A significância estatística encontrada para a função de mastigação, visto que foi com padrão inesperado, pode ter ocorrido em virtude do restrito número de sujeitos em cada subgrupo.

Assim, sugerem-se pesquisas futuras, com um número maior de sujeitos, para complementar este estudo.

\begin{abstract}
Purpose: to investigate the relation between time and type of feeding with chewing, swallowing and breathing functions. Method: the sample consisted of 52 children of both genders, aged between 5 and 8 year-old. Through a questionnaire, we collected data regarding type and duration of children's breastfeeding and breathing. The children were submitted to clinical evaluation of functions, based on the Orofacial Myofunctional Assessment Protocol with Expanded Scores (AMIOFE-E). Data were descriptively and statistically analyzed. For testing the normality of the quantitative variable, we used the Lilliefors test. All relations among the variables were checked through Chi-square test, considering a 5\% significance level. Results: there was no statistically significant relation between duration and type of feeding and breathing and swallowing variables. There was a statistical significance between feeding type and chewing, but not between feeding duration and this variable. Furthermore, the significant relation failed to follow the expected pattern. Conclusion: it was possible to conclude that, generally, there was no significant relation between time and type of feeding and the investigated functions, except for the significance between type of feeding and chewing. Probably, the lack of significance is due to the fact that the development of all the stomatognathic system has multifactorial sources. The statistical significance found as for the chewing function, since it showed an unexpected pattern, may have occurred because of the restricted number of subjects in each subgroup.
\end{abstract}

KEYWORDS: Stomatognathic System; Mouth Breathing; Breastfeeding; Chewing; Deglutition

\section{REFERÊNCIAS}

1. Green JR, Moore CA, Higashikawa M, Steve RW. The physiologic development of speech motor control: lip and jaw coordenation. J. Speech Lang Hear Res. 2000;43:239- 55.

2. Casagrande L, Ferreira FV, Hahn D, Unfer DT, Praetzel JR. Aleitamento natural e artificial e o desenvolvimento do sistema estomatogmático. Rev. Fac. Odontol. maio/ago, 2008; 49(2): 11- 7.

3. Junqueira P. Amamentação, hábitos orais e mastigação: orientações, cuidados e dicas. $3^{\underline{a}}$ ed. Rio de Janeiro: Revinter; 2005.

4. World Health Organization. The optimal duration of exclusive breastfeeding: a systematic review. Geneva: WHO; 2001.

5. Brasil. Ministério da Saúde. Guia Alimentar para Crianças Menores de 2 anos de Idade. Brasília. 152; 2002.

6. Vieira GO, Silva LR, Almeida JAG, Cabral VA. Hábitos alimentares de crianças menores de 1 ano amamentadas e não amamentadas. J Pediatr. 2004; 80:411-6.
7. Rea MF. O pediatra e a amamentação exclusiva. J Pediatr. 2003;79:479-80.

8. Medeiros APM, Ferreira JTL, Felício CM. Correlação entre métodos de aleitamento, hábitos de sucção e comportamentos orofaciais. Pró-Fono Rev Atual Cient. out-dez, 2009; 21(4):315-9.

9. Carrascoza KC, Possobon RF, Tomita LM, Moraes ABA. Consequences of bottle-feeding to the oral facial development of initially breastfed children. J Pediatr. 2006; 82: 395-7.

10. Neiva FCB, Catonni DM, Ramos JLA, Isller $\mathrm{H}$. Desmame precoce: implicações para o desenvolvimento motor-oral. J Pediatr. 2003; 79(1):07-12.

11. Felício CM, Folha GA, Ferreira CLP, Medeiros APM . Expanded protocol of orofacial myofunctional evaluation with scores: Validity and reliability. International Journal of Pediatric Otorhinolaryngology. 2010; 74(11):1230-9.

12. Padovan BAE. Deglutição atípica. Separata Reeducação mioterápica nas pressões atípicas da língua. Ortodontia. 1976; 9(1-2):5-59. 
13. Vaucher ALI, Durman S. Amamentação: crenças e mitos. Rev Eletr Enf. 2005; 07: 207-14.

14. Heringer MRC, Reis M, Pereira LFS, Di Ninno CQMS. A influência da amamentação natural no desenvolvimento dos hábitos orais. Rev CEFAC. Jul-set, 2005; 7(3): 307-10.

15. Oliveira AB, Souza FP, Chiappetta ALML. Relação entre hábitos de sucção não-nutritiva, tipo de aleitamento e má oclusões em crianças com dentição decídua. Rev CEFAC. jul-set, 2006; 8(3): 352-9.

16. Carvalho GD. A amamentação sob a visão funcional e clínica da odontologia. Revista Secretários de Saúde. 1995;10:12-3.

17. Leite ICG, Rodrigues CC, Faria AR, Medeiros GV, Pires LA. Associação entre aleitamento materno e hábitos de sucção não nutritivos. Revista da Associação Paulista dos Cirurgiões Dentistas. 1999; 53:151-5.

18. Barbosa C, Schnonberger MB. Importância do aleitamento materno no desenvolvimento da motricidade oral. In: Marchesan IQ, Zorzi JL, Gomes IC, eds. Tópicos em Fonoaudiologia. São Paulo: Lovise; 1996. 435-46.

19. Hitos SF, Periotto MC. Amamentação - Atuação Fonoaudiológica - Uma Abordagem Prática e Atual. Rio de Janeiro: Revinter, 2009.

20. Nicolielo AP, Gross C, Berretin-Felix G, Machado MAMP. Fatores interferentes na alimentação de crianças de 17 a 25 meses de uma creche municipal. Rev. CEFAC. 2009; 11(3);291-7.
21. Trawitzki LVV, Anselmo-Lima WT, Melchior MO, Grechi TH, Valera FCP. Aleitamento e hábitos orais deletérios em respiradores orais e nasais. Rev Bras Otorrinolaringol. No/dez, 2005; 71 (6): 747-51.

22. Diaz Morell JE, Farinas Cordon MM, Pellitero Reyes BL, Alvarez Infante E. La respiracion bucal y su efecto sobre la morfologia dentomaxilofacial. Correo Cientifico Medico de Holguin. [periódico na Internet]. 2005; [acesso em 23/05/2011]; 9(1). Disponível em: http://www.cocmed.sld.cu/no91/ n91ori6.htm.

23. Felício CM, Melchior MO, Silva MAMR, Celeghini RMS. Desempenho mastigatório em adultos relacionado com a desordem temporomandibular e com a oclusão. Pró-Fono Rev Atual Cient. abr-jun, 2007; 19 (2): 151-8.

24. Bicalho GP, Motta ARM, Vicente LCC. Avaliação da deglutição em crianças respiradoras orais. Rev CEFAC. 2006; 8(1):50-5.

25. Imbaud T, Wandalsen G, Nascimento Filho E, Wandalsen NF, Mallozi MC, Solé D. Respiração bucal em pacientes com rinite alérgica: fatores associados e complicações. Rev Bras Alerg Imunopatol. 2006; 29(4):183-7.

26. Marchesan IQ. Avaliação e Terapia dos Problemas da Respiração. In: Fundamentos em Fonoaudiologia - Aspectos Clínicos da Motricidade Oral. $2^{\mathrm{a}}$ ed. Rio de Janeiro: GuanabaraKoogan; 2005. p. 23-36.

http://dx.doi.org/10.1590/S1516-18462012005000020

RECEBIDO EM: 14/06/2011

ACEITO EM: 31/08/2011

Endereço para correspondência:

Aline Prade Neu

Rua Doutor Bozano, 1094 ap. 06

Santa Maria - RS

CEP: 97015-002

E-mail: alineprade@gmail.com 